\title{
Nicotine exposure during adolescence alters the rules for prefrontal cortical synaptic plasticity during adulthood
}

\author{
Natalia A. Goriounova and Huibert D. Mansvelder* \\ Department of Integrative Neurophysiology, CNCR, Neuroscience Campus Amsterdam, VU University, Amsterdam, Netherlands
}

\section{Edited by:}

Darwin K. Berg, University of

California, San Diego, USA

\section{Reviewed by:}

William N. Green, University of Chicago, USA

Peter B. Sargent, University of California, San Francisco, USA

\section{${ }^{*}$ Correspondence:}

Huibert D. Mansvelder, Department of Integrative Neurophysiology, Center for Neurogenomics and Cognitive Research (CNCR),

Neuroscience Campus Amsterdam, VU University Amsterdam,

De Boelelaan 1085, Room C-440,

1081 HV Amsterdam, Netherlands. e-mail: huibert.mansvelder@

cncr.vu.nl
The majority of adolescents report to have smoked a cigarette at least once. Adolescence is a critical period of brain development during which maturation of areas involved in cognitive functioning, such as the medial prefrontal cortex (mPFC), is still ongoing. Tobacco smoking during this age may compromise the normal course of prefrontal development and lead to cognitive impairments in later life. In addition, adolescent smokers suffer from attention deficits, which progress with the years of smoking. Recent studies in rodents reveal the molecular changes induced by adolescent nicotine exposure that alter the functioning of synapses in the PFC and underlie the lasting effects on cognitive function. In particular, the expression and function of metabotropic glutamate receptors (mGluRs) are changed and this has an impact on short- and long-term plasticity of glutamatergic synapses in the PFC and ultimately on the attention performance. Here, we review and discuss these recent findings.

Keywords: adolescence, nicotine, prefrontal cortex, STDP, mGluR, nAChR, cognitive behavior
Adolescence is an important developmental period when a child has to make a transition to an independent status of adult. Such transition demands an ability to take risks and a taste for novelty but also emergence of self-control and more adult decisionmaking strategies. Also, brain development is not complete by adolescence, especially maturation of areas involved in cognitive functioning, such as the medial prefrontal cortex (mPFC), is still ongoing. Brain development continues throughout adolescence, though the speed and timing of maturation varies for different brain areas (Gogtay et al., 2004). Subcortical limbic structures important for emotional processing, such as hypothalamus, midbrain dopamine areas, nucleus accumbens, dorsal, and ventral striatum and amygdala, experience a major developmental boost around the onset of puberty (Sowell et al., 2003; Casey et al., 2005). Their maturation is important for social and sexual behaviors and is triggered by pubertal hormones. In contrast, development of frontal cortical areas of the brain, responsible for cognitive control over behavior, depends on age and experience and continues throughout adolescence and into adulthood (Sowell et al., 2003; Giedd, 2004). Thus, during adolescence emotional drive has already become very strong while cognitive self-control and adult decision-making strategies still are developing. Thereby, brain development may be responsible for characteristic adolescent traits-uncontrollable mood swings, impulsivity, risk-taking, and peer-directed social interactions (Orr and Ingersoll, 1995; Spear, 2000; Galvan et al., 2007). Although indispensable for transition from child to independent status of adult, these traits hold hazards. Indeed, risktaking behavior, so typical for adolescents, is associated with high rates of mortality and morbidity among young people (Grunbaum et al., 2004).
The impulsive, peer-influenced nature of adolescent choices sets the stage for experimenting with drugs of abuse. Since nicotine is one of the most socially accepted drugs in our society, the first choice usually falls on tobacco smoking. According to a recent study conducted in 41 countries in Europe and North America, 19\% of 15-year olds smoke at least once a week and $30 \%$ report experimenting with cigarettes before the age of 14 (Currie et al., 2008). Serious health risks of smoking are wellknown: smoking leads to millions of premature deaths worldwide and tobacco smoking has been marked as an epidemic disease (Peto et al., 1999). Nicotine is also a psychoactive and addictive substance that directly acts on brain areas involved in emotional and cognitive processing. Early exposure to nicotine during adolescence may disturb the normal course of brain maturation and have lasting consequences for cognitive ability, mental health, and even personality (Brown et al., 1996; Choi et al., 1997; Richards et al., 2003; Brook et al., 2004; Deas, 2006). In humans, the PFC shows delayed development with respect to other cortical areas during adolescence with delayed thinning of cortical grey matter, most likely reflecting fine-tuning of synaptic contacts (Gogtay et al., 2004; Sowell et al., 2004; Casey et al., 2005). Rearrangement of local inhibitory inputs and decreases in synaptic densities and branch points of excitatory connections between pyramidal neurons occur within the developing PFC (Woo et al., 1997; Cruz et al., 2003). Spike-timing-dependent modifications are likely to be important for cortical development, map plasticity, and functioning of neural networks: correlated inputs lead to strengthening of connections (LTP) while uncorrelated inputs lead to weakening (LTD) and pruning of unused synapses (Bi and Poo, 2001; Song and Abbott, 2001; Feldman and Brecht, 2005). Here, we highlight recent findings that start to uncover 
causal relations between nicotine exposure during adolescence and cognitive deficits in later life, with an emphasis on synaptic adaptations and altered rules for synaptic plasticity in prefrontal networks.

\section{IMMEDIATE EFFECTS OF NICOTINE EXPOSURE}

Nicotine activates nicotinic acetylcholine receptors (nAChR), which take part in cholinergic signalling. Twelve genes have been identified encoding neuronal nicotinic receptors (for review see Le Novere et al., 2002; Millar and Gotti, 2009). In the central nervous system $9 \alpha$-subunits $(\alpha 2-\alpha 10)$ and $3 \beta$-type subunits $(\beta 2-\beta 4)$ are expressed. These subunits assemble in different stoichiometries to form pentameric channels, and subunit compositions of $\mathrm{nAChRs}$ vary depending on the brain region (for review see Grady et al., 2002; Le Novere et al., 2002; McGehee, 2002; Alkondon and Albuquerque, 2004; Wonnacott et al., 2005; Mineur and Picciotto, 2008; Millar and Gotti, 2009). Nicotinic AChRs are cation selective channels that permit the flow of $\mathrm{Na}^{+}$, $\mathrm{K}^{+}$, and $\mathrm{Ca}^{2+}$ across the membrane, which leads to depolarizing currents and activate neurons (McGehee and Role, 1995; Millar and Gotti, 2009).

Modulation of PFC activity by nAChRs will depend on which cell types express nAChRs and what subunits they are made of. In the PFC, nAChR expression is found across all layers (Gioanni et al., 1999; Poorthuis et al., 2012). Activation of nAChRs can alter pyramidal neuron activity by two mechanisms: presynaptically enhancing glutamatergic inputs or by activating postsynaptic receptors directly (Poorthuis et al., 2012). Recently we measured nAChR activation by making whole-cell recordings from PFC pyramidal neurons in the different layers and used wild-type, $\beta 2$-null, or $\alpha 7$-null mice as well as pharmacological tools to determine the nAChR subunits involved. We showed that PFC pyramidal neurons across cortical layers show a differential pattern of postsynaptic nAChR activation: layer II/III pyramidal neurons do not contain nAChRs, layer $\mathrm{V}$ pyramidal neurons contain $\alpha 7 \mathrm{nAChRs}$, and layer VI pyramidal neurons are modulated by $\beta 2 *$ nicotinic receptors (Poorthuis et al., 2012). Also presynaptic glutamatergic inputs can be modulated by nicotine (Gioanni et al., 1999; Lambe et al., 2003; Couey et al., 2007; Poorthuis et al., 2012). We found that this presynaptic regulation is specific to layer $\mathrm{V}$, only moderately present in layer VI and not present in layer II-III (Poorthuis et al., 2012).

In addition to direct activation of PFC pyramidal neurons by nAChRs, PFC GABAergic interneurons across all layers are also directly activated by $\mathrm{nAChR}$ stimulation (Poorthuis et al., 2012). Increased inhibition through activation of nAChRs expressed by interneurons has been found in many different brain regions (Jones and Yakel, 1997; Xiang et al., 1998; McQuiston and Madison, 1999; Alkondon et al., 2000; Ji and Dani, 2000; Mansvelder et al., 2002; Gulledge et al., 2007). Interneurons form a highly diverse group of cells with distinct roles in cortical computation (Kawaguchi, 1993; Markram et al., 2004). Fastspiking cells target the perisomatic region of pyramidal neurons (Kawaguchi and Kubota, 1997; Kawaguchi and Kondo, 2002) and are therefore thought to be involved in regulating the activity window of pyramidal neurons. Feedforward inhibition in the PFC plays an important role in the integration of hippocampal inputs, which enter the PFC through superficial layers (Jay and Witter, 1991; Tierney et al., 2004). Fast-spiking cells in PFC layer II-III contain $\alpha 7$ nAChRs, as do as about half of the fast-spiking cells in layer V (Poorthuis et al., 2012). nAChR activation on fastspiking interneurons in PFC layer II/III may alter processing of hippocampal inputs.

Somatostatin-positive cells target distal dendritic regions (Kawaguchi and Kondo, 2002; Silberberg and Markram, 2007) and can mediate disynaptic inhibition between pyramidal neurons (Silberberg and Markram, 2007; Kapfer et al., 2007). Regular-spiking and somatostatin-positive cells in PFC layer IIIII and $\mathrm{V}$ are positive for $\mathrm{nAChRs}$, suggesting that $\mathrm{nAChRs}$ play an important role in modulating feedback inhibition among pyramidal neurons in these layers (Poorthuis et al., 2012).

Finally, we showed that the network activity in the PFC in response to bath application of ACh is dominated by $\beta 2 \mathrm{nAChRs}$ activation. Receptors of this subtype are expressed by pyramidal neurons in layer VI, glutamatergic inputs to layer V and VI and by interneurons in all layers of the PFC. In summary, $\beta 2$ containing $\mathrm{nAChRs}$ stimulate both excitatory and inhibitory neurons in deep layers, while in layer II/III only interneurons are activated (Poorthuis et al., 2012). Thus, the net result of nicotinic receptor stimulation is the increased inhibitory transmission in superficial PFC layers, whereas in deep layers it also leads to activation of pyramidal neurons. This pattern of activation alters the information processing in prefrontal networks and can directly alter rules for plasticity (Couey et al., 2007).

One of the forms of long-term plasticity, spike-timing dependent plasticity (STDP), is based on Hebb's postulate often summarized as "Cells that fire together, wire together" (Hebb, 1949). It attempts to explain associative learning, in which nearly simultaneous activation of cells increases the strength of synaptic contacts between those cells. This type of plasticity is thought to play an important role in cortical development during critical stages and underlie some forms of learning (Feldman et al., 1999; Feldman and Brecht, 2005; Letzkus et al., 2007). In PFC, STDP can be directly modulated by nicotine (Couey et al., 2007; Goriounova and Mansvelder, 2012). We showed that in adolescent rats, STDP in layer $\mathrm{V}$ pyramidal neurons is strongly reduced if nicotine is applied to PFC slices during induction protocol (Goriounova and Mansvelder, 2012). In PFC layer V, the increase in inhibition dominates the effects of nicotine on synaptic plasticity (Couey et al., 2007). Nicotine-stimulated inhibition reduces dendritic calcium signaling and renders postsynaptic activity in layer $\mathrm{V}$ pyramidal cells insufficient to induce potentiation (Couey et al., 2007). In layer II/III, the layer where the synaptic inputs were stimulated to induce STDP, nAChR activation results predominantly in activation of interneurons (Poorthuis et al., 2012). Thus, nicotine-induced increase in inhibitory transmission can explain the decrease in spike-timing dependent potentiation in adolescent rats.

\section{UPREGULATION OF nAChR AND SYNAPTIC mGIuR EXPRESSION}

Adolescents may be more vulnerable to nicotine addiction due to greater positive effects nicotine has on adolescents than adults, whereas the negative effects associated with nicotine, such as 
withdrawal are smaller in adolescents (O'Dell, 2009). Nicotine administration during, but not following adolescence, has longlasting effects on cognitive, addictive, and emotional behavior in rats (Adriani et al., 2003; Iniguez et al., 2008; Counotte et al., 2009, 2011). Furthermore, adolescent animals are more sensitive to nicotine conditioned place preference than adults and show this at lower nicotine doses (Vastola et al., 2002; Belluzzi et al., 2004; Shram et al., 2006; Brielmaier et al., 2007; Kota et al., 2009). Adolescent nicotine exposure leads to acute and longer-lasting changes in nAChR binding (Abreu-Villaca et al., 2003; Doura et al., 2008) and function (Kota et al., 2009) in brain regions such as cortex and striatum. We recently found that the adolescent rodent brain is more sensitive to nicotinic receptor upregulation in the medial PFC (mPFC) than adults (Counotte et al., 2012). Naïve rats show an age-related decrease in ${ }^{3} \mathrm{H}$-epibatidine labeled high-affinity nicotinic receptors in the $\mathrm{MPFC}$, but not in occipital cortex. Adolescent, but not adult nicotine exposure increases ${ }^{3} \mathrm{H}$-Epi-binding of $\mathrm{mPFC}$ receptors on the first day of abstinence following 10 days of nicotine injections. This is paralleled by an $\mathrm{mPFC}$-specific increase in expression of $\mathrm{nAChRs}$ containing $\alpha 4$ and $\beta 2$ (but not $\alpha 5$ ) subunits or high-affinity nAChRs. The increased expression of high-affinity nAChRs in adolescents is accompanied by an increase in nicotine-stimulated GABAergic synaptic transmission in the mPFC (Counotte et al., 2012).

One of the first and most common cellular adaptations following chronic nicotine exposure is the upregulation of nicotinic receptor levels (Dani and Bertrand, 2007). Especially $\alpha 4 \beta 2$ type of nAChRs appears to be selectively upregulated via posttranslational mechanisms (Miwa et al., 2011). The upregulation of $\alpha 4 \beta 2$ nAChRs by chronic nicotine treatment has been replicated many times in numerous systems - transfected cell lines, neurons in culture, brain slices, and smokers' brains (Wonnacott, 1990; Fu et al., 2009; Lester et al., 2009; Marks et al., 2011; Miwa et al., 2011). Upregulation is not accompanied by an increase in nAChR subunit mRNA (Marks et al., 1992), instead it leads to increased $\mathrm{nAChR}$ protein levels resulting from posttranslational mechanisms (Govind et al., 2009; Marks et al., 2011). According to one view on nAChR upregultation, nicotine acts intracellularly as a selective pharmacological chaperone of acetylcholine receptor (Lester et al., 2009). It stabilizes nAChRs during assembly and maturation and this stabilization is most pronounced for the high-affinity $\mathrm{nAChR}$ containing $\alpha 4 \beta 2$ subunits. Other possible mechanisms underlying $\mathrm{nAChR}$ upregulation can result from increased cell surface turnover, increased receptor trafficking to the surface, changes in subunit stoichiometry or nAChR conformational changes (Govind et al., 2009). All these proposed mechanisms have two features in common: they are posttranslational and involve upregulation of high-affinity $\mathrm{nAChR}$ containing $\alpha 4 \beta 2$ subunits. Indeed, we found that specifically high-affinity nicotinic receptors containing the $\alpha 4$ and $\beta 2$ subunits were upregulated in the adolescent PFC shortly following nicotine exposure. This upregulation was paralleled by a functional elevation in nicotine-stimulated GABAergic transmission, indicating that functional surface $\mathrm{nAChRs}$ are upregulated as well (Counotte et al., 2012).

Given that pyramidal neurons and excitatory projections in layer II/III of the PFC do not express nAChRs (Poorthuis et al.,
2012), the functional consequence of $\alpha 4 \beta 2$ nAChR upregulation on interneurons in layer II/III will be an increased inhibitory transmission in superficial PFC layers. In the deep layers of the PFC, $\beta 2$ subunits are expressed by both interneurons as well as layer VI pyramidal neurons and excitatory inputs to layer $\mathrm{V}$ pyramidal neurons. An upregulation of these receptors will lead to a combined increase in activation of pyramidal neurons and interneurons. Since $\beta 2$-containing $\mathrm{nAChRs}$ in the $\mathrm{mPFC}$ control attention performance (Guillem et al., 2011) this may have functional implications for maturation and function of the prefrontal network. During chronic nicotine exposure of the adolescent PFC, the pattern of activity in prefrontal network may gradually shift toward activation of excitatory neurons in deep layers in the context of increased overall inhibition. This may affect plasticity and refinement of cortical connections (Couey et al., 2007), even though GABAergic transmission by itself was not affected directly following nicotine treatment during adolescence (Counotte et al., 2012). Still, we find that also directly following nicotine exposure during adolescence in vivo, STDP of glutamatergic synapse strength is blocked (Goriounova and Mansvelder, 2012). Since in these experiments assessing STDP immediately following nicotine treatment during adolescence nicotine was not applied, this can not explain the reduced STDP we observed.

How is spike-timing-dependent potentiation affected by nicotine exposure during adolescence? In addition to an upregulation of nAChRs, we recently found in a large-scale iTRAQbased proteomics screen of synaptic protein levels in the PFC that metabotropic glutamatergic receptors type 2 (mGluR2) are significantly upregulated during adolescent nicotine exposure (Figure 1) (Counotte et al., 2011). This short-term upregulation is followed by a long-term decrease in mGluR2 levels 5 weeks after the nicotine exposure during adolescence. As will be discussed later, this pattern of mGluR2 expression leads to opposing effects on glutamatergic transmission and plasticity in PFC.

Metabotropic GluR2s are located presynaptically on glutamatergic synapses and their activation reduces the probability of glutamate release. Thereby, an upregulation of mGluR2 levels diminishes activity of excitatory glutamatergic synapses in the PFC. Thus, increases in functional nAChR on inhibitory neurons and increased nicotine-stimulated excitation in deep layers of the PFC may be counteracted by reduced excitatory synaptic activity mediated by increased mGluR2 activity. Blocking mGluR2s with MPPG restored spike-timing-dependent potentiation following nicotine exposure during adolescence back to levels observed in animals that received saline treatment during adolescence (Goriounova and Mansvelder, 2012). This suggests that the upregulation of presynaptic mGluR2s after nicotine exposure during adolescence alters the rules for STDP in PFC networks.

In summary, the net result of nicotine in the short-term in adolescent $\mathrm{mPFC}$ that is chronically exposed to nicotine, very likely amounts to persistently enhanced levels of inhibition across all cortical layers possibly combined with some increase in thalamocortical glutamate release by presynaptic (or preterminal) nAChRs in deep layers. The latter effect, however, is most likely counteracted by elevated presynaptic mGluR2 levels. This general increase in inhibition plays a role in the plasticity and refinement of cortical connections (reduced STDP in nicotine) and thus 


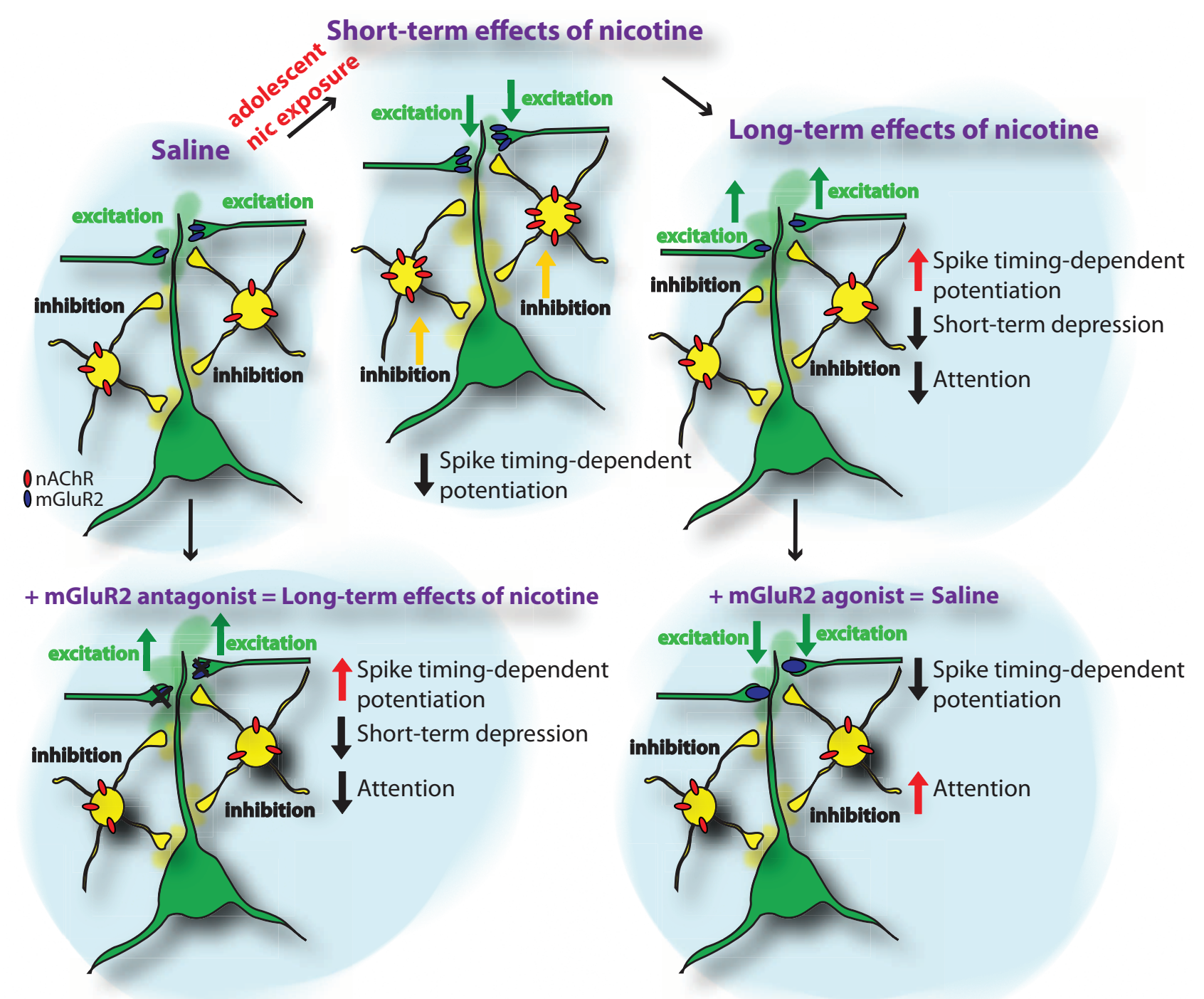

FIGURE 1 | Schematic representation of the short-term and long-term adaptations in PFC neuronal networks caused by nicotine exposure during adolescence. The upper panels show the sequence of adaptations in $\mathrm{nAChR}$ and mGluR2 protein levels and the resulting changes in inhibition and excitation and attention behavior from control conditions (saline) to nicotine exposure during adolescence (short-term effects of nicotine) and 5 weeks following nicotine exposure (long-term effects of nicotine). The lower panels show the effects of mGluR2 agonists and antagonists in saline and nicotine-exposed animals. Applying mGluR2 antagonists to the adult mPFC reduces depression of glutamatergic synapses and reduces attention performance of the animal. Providing mGluR2 agonists to the mPFC of adult rats that were exposed to nicotine during adolescence normalizes synaptic depression and spike-timing-dependent potentiation of glutamatergic synapses and improves attention performance. may have functional implications for cognitive processing and maturation of prefrontal network.

\section{LONG-TERM CONSEOUENCES}

Smoking during adolescence is associated with disturbances in working memory and attention as well as reduced PFC activation (Jacobsen et al., 2005, 2007; Musso et al., 2007). Smoking is also a prospective risk factor for impaired cognitive function in later life; heavy smoking predicts incident cognitive impairment and decline (Cervilla et al., 2000; Kalmijn et al., 2002; Richards et al., 2003). In animal studies exposure during adolescence induces stronger changes in gene expression in the PFC than during other periods of development and adulthood (Schochet et al., 2005, 2008; Polesskaya et al., 2007). The adolescent PFC shows nicotine response in gene regulation involved in vesicle release, signal transduction, cytoskeleton dynamics, and transcription, suggesting the role of chronic nicotine exposure in initiating long-term structural and functional adaptations (Polesskaya et al., 2007). The expression of key molecules involved in plasticity is also altered in the PFC by adolescent nicotine exposure. Acute nicotine induces increases in the expression of the dendritically targeted dendrin mRNA in PFC of adolescent but not adult animals. Dendrin is an important component of cytoskeletal modifications at the synapse and therefore can lead to unique plasticity changes 
in the adolescent PFC (Schochet et al., 2008). Lasting synaptic adaptations involve activation of intracellular signaling pathways and enzymes such as extracellular regulated protein kinase (ERK) and cAMP response element binding protein (CREB). Specifically in the PFC, increases in phosphorylation of both these enzymes were found after repeated nicotine exposure (Brunzell et al., 2003). Also changes in macromolecular constituents indicative of cell loss (reduced DNA) and altered cell size (protein/DNA ratio) can be seen in cortical regions of rodents after adolescent nicotine treatment (Trauth et al., 2000). Further, repeated nicotine exposure also alters the structure of neurons in mPFC: it increases both dendritic length and spine density (Brown and Kolb, 2001). Long-term changes have been observed in dendritic morphology of specific subpopulations of pyramidal neurons and these structural changes depended on the age of drug exposure (Bergstrom et al., 2008).

Also on the behavioral level, nicotine during adolescence leads to persisting deficits. Adolescent, but not adult, nicotine treatment reduces accuracy of correct stimulus detection in a visuospatial attentional task, with an increase in premature and time-out responding that suggests impaired attention and lack of impulsive control which is part of normal adolescent maturation (Counotte et al., 2009). Similar nicotine-induced deficits have been found in a serial pattern learning paradigm (Fountain et al., 2008). In a recent study, chronic nicotine exposure during adolescence produced long-lasting impairments in contextual learning that were observed during adulthood, whereas adult chronic nicotine exposure did not (Portugal et al., 2012).

Taken together, these studies in rodents show that nicotine exposure during adolescence induces significant changes in gene expression, neuronal morphology, and behavior in PFC. Thus, nicotine does not only change cholinergic signalling by altering nicotinic receptor levels in the adolescent PFC, but can also lead to secondary adaptations involving structural and functional changes in cognition. What are the changes that underlie the changes in cognitive performance?

\section{LASTING SYNAPTIC ADAPTATIONS IN THE PFC}

In adult rodents that were exposed to nicotine during adolescence only a handful of proteins show long-term adaptations following adolescent nicotine exposure that persisted into later life. Nicotinic AChR levels in the PFC returned to baseline 5 weeks following adolescent nicotine exposure (Counotte et al., 2012). In contrast, mGluR2 levels show a strong down-regulation at this time (Counotte et al., 2011). Reduced mGluR2 function in $\mathrm{mPFC}$ synapses resulted in impaired attention performance. Stimulating mGluR2s with specific agonists improved attention performance in animals that were exposed to nicotine during adolescence (Counotte et al., 2011). Interestingly, the association between changes in mGluR2 signalling and nicotine exposure is not limited to the PFC. Also in other brain areas involved in reward processing such as ventral tegmental area (VTA) and the nucleus accumbens (NAcc) lasting adaptations in mGluR2 function follow nicotine exposure and were found to affect rewarding properties of nicotine (Helton et al., 1997; Kenny et al., 2003; Kenny and Markou, 2004; Liechti et al., 2007). In these brain areas, activation of $\mathrm{mGlu} 2 / 3$ receptors decreases nicotine self-administration (Liechti et al., 2007), and they play an important role in the development of drug dependence and the expression of the negative affective state observed during withdrawal (Pilc et al., 2008). However, the role of group II mGlu receptors in withdrawal appears complex and most likely depends on changes in multiple brain areas.

Although the sequence of events linking mGluR2 adaptations to $\mathrm{nAChR}$ activation is unknown, it seems that the reasons for its up- and down-regulation pattern after adolescent nicotine exposure may lie in its function. Metabotropic GluR2 receptors are located on presynaptic glutamatergic terminals where they are activated by glutamate spill over to inhibit glutamate release (Mateo and Porter, 2007). It was shown that activation of mGluR2s can also regulate release of other neurotransmitters: it can inhibit GABA release via a presynaptic mechanism (Bradley et al., 2000; Pilc et al., 2008). Given the inhibitory role of mGluR2 in neurotransmitter release, its function seems to counteract that of nAChR, which enhances both excitatory and inhibitory synaptic transmission (Lambe et al., 2003; Couey et al., 2007; Poorthuis et al., 2012). The short-term effects of adolescent nicotine exposure most likely involve enhanced levels of inhibition in prefrontal network. Accordingly, we found an initial and transient upregulation of inhibitory mGluR2 receptor directly following nicotine exposure during adolescence (Counotte et al., 2011), which would contribute to the same effect.

In general, factors that lead to enhanced excitation can cause alterations in mGluR2 transmission and cause cognitive deficits (Melendez et al., 2004; Pozzi et al., 2011). Enhanced glutamate release in PFC was found to be associated with attention deficit and loss of impulse control (Pozzi et al., 2011). MGluR2 agonists are effective in improving cognitive deficits if enhanced glutamate release is caused by NMDA receptor antagonists (Pozzi et al., 2011). Furthermore, the important role of prefrontal mGluR2 signaling in cognition is stressed by its link to brain disorders such as depression and schizophrenia. Activation of this receptor has even been proposed as a novel treatment approach for these disorders (Gupta et al., 2005; Palucha and Pilc, 2005; Pilc et al., 2008; Conn et al., 2009). Thus, mGluR2 signalling seems to be a good candidate for shaping cognitive behavior and its impairment leads to disturbances in cognitive function.

At the level of synapse function, alterations in mGluR2 levels affect both short-term synaptic plasticity as well as STDP in later life. Short-term depression (STD) is reduced in adult animals as a result of nicotine exposure during adolescence (Counotte et al., 2011). In control animals, blocking mGluR2 signalling with mGluR2 antagonists also results in reduced STD. Reduced mGluR2 signalling after nicotine exposure has a similar effect on STD as mGluR2 block by antagonist (Figure 1). Thereby, mGluR2 may act as an inhibitory feedback mechanism in conditions of excessive excitation and high glutamate release, as occurs when a neuron fires a train of action potentials. Especially at high frequency stimulation the effect of mGluR2 on STD was most prominent at excitatory synapses on layer $\mathrm{V}$ pyramidal neurons in the PFC (Counotte et al., 2011). The lasting reduction of mGluR2 levels and function after adolescent nicotine exposure leads to reduced inhibitory feedback on pyramidal cells and reduces the regulatory role of this receptor in short-term 
plasticity. Most likely, activation of mGluR2s affects presynaptic calcium channel function as was found in the calyx of Held, by direct electrophysiological recordings from presynaptic terminals (Takahashi et al., 1996). Agonists of metabotropic glutamate receptors (mGluRs) suppressed high voltage-activated P/Q-type calcium channels in the presynaptic terminal, thereby inhibiting transmitter release (Takahashi et al., 1996). Since presynaptic $\mathrm{Ca}^{2+}$ dynamics play a key role in short-term plasticity (Zucker and Regehr, 2002), decrease in $\mathrm{Ca}^{2+}$ current may explain mGluR-dependent modulation of STD.

STD may equip the synapse with low-pass filtering properties, by which the synapse will pass on the first of stimulus in a train of stimuli unaltered, while the rest are attenuated. In this manner it shapes the information transfer by synaptic networks and gives rise to sensory and behavioral phenomena (Zucker, 1989). For example, in somatosensory cortex of rat, in vivo whole-cell recordings in cortical neurons during whisker deflection directly demonstrated that synaptic depression of thalamic input to the cortex contributes to rapid adaptation of sensory responses (Chung et al., 2002). Selective attention, the ability of an organism to filter out relevant information in the face of distractors, can build upon just such synaptic process. Layer $\mathrm{V}$ pyramidal neurons in PFC handle diverse incoming information from mediodorsal thalamus and from local neurons and these connections are important in mediating executive functions such as for example working memory (Floresco et al., 1999). STD on this level may represent a higher level of sensory adaptation that can be expressed as decreased levels of attention and responsiveness. Reduced short-term plasticity after nicotine exposure compromises the ability of prefrontal neurons to efficiently filter out irrelevant information.

We recently found that glutamatergic synapses in the PFC show increased spike-timing-dependent LTP 5 weeks after nicotine exposure during adolescence (Figure 1) (Goriounova and Mansvelder, 2012). This was not the case when animals were exposed to nicotine during adulthood, indicating that adolescence is a vulnerable period for these lasting changes to occur. The long-term effects on LTP 5 weeks after nicotine exposure during adolescence were opposite to the effects immediately following nicotine exposure during adolescence, where spiketiming-dependent LTP is supressed. Thus, nicotine exposure during adolescence has lasting effects on the mechanisms of STDP and persistent synaptic alterations that increase LTP.

What is the mechanism underlying the long-term effects of nicotine exposure during adolescence on STDP? We hypothesized that altered levels of mGluR2 receptors can explain the lasting effects on STDP. Reduced mGluR2 signalling in adult rats after nicotine exposure (Counotte et al., 2011) may contribute to the decreased plasticity we observed. Blocking mGluR2 receptors with MPPG resulted in increased LTP comparable to levels observed in adult rats treated with nicotine during adolescence (Goriounova and Mansvelder, 2012). In nicotinetreated rats, where the synaptic mGluR2 receptor levels are reduced (Counotte et al., 2011), enhancing mGluR2 activity by applying mGluR2 agonist LY379268 completely abolished LTP (Goriounova and Mansvelder, 2012). Thereby, mGluR2 signalling bidirectionally influences spike-timing-dependent LTP: reducing mGluR2-dependent inhibition leads to increased LTP, while enhancing mGluR2 activation blocked LTP. Thus, immediately following adolescent nicotine exposure, increased levels of mGluR2s may be responsible for reduced LTP induction, and 5 weeks following adolescent nicotine exposure, the lasting reduction in mGluR2 signalling can explain the increased LTP in the adult mPFC.

STDP depends on the precise timing of the synaptic input and the postsynaptic action potential and this temporal relationship resembles typical features of associative learning (Letzkus et al., 2007). Although STDP has not been directly linked to attention performance, the ability to associate goal-relevant information is crucial for any cognitive behavior. In nicotine-treated rats the same amount of pre- and postsynaptic activity leads to more synaptic potentiation. This may suggest that the PFC network would even associate irrelevant stimuli.

\section{CONCLUSION}

The prefrontal cortex, the brain area responsible for executive functions and attention performance, is one of the last brain areas to mature and is still in the process of developing during adolescence. This places the adolescent brain in a vulnerable state of imbalance, susceptible to the influence of psychoactive substances such as nicotine. In prefrontal networks nicotine modulates information processing on multiple levels by activating and desensitizing nicotine receptors on different cell types and in this way affects cognition. The adolescent brain is particularly sensitive to the effects of nicotine. Studies in human subjects indicate that smoking during adolescence increases the risk of developing psychiatric disorders and cognitive impairment in later life. In addition, adolescent smokers suffer from attention deficits, which progress with the years of smoking.

From studies in the rodent brain it is becoming clear that on the short-term, adolescent, but not adult, nicotine exposure increases the expression of nAChRs containing $\alpha 4$ and $\beta 2$ subunits in the $\mathrm{mPFC}$, which leads to an increase in nicotine-induced GABAergic synaptic transmission. In addition, mGluR2 levels on presynaptic glutamatergic terminals in the PFC are increased, causing a reduction in glutamatergic synapse strength and reducing STDP (Figure 1). Changes in nAChR levels are reversible: in the adult rodent brain, weeks after nicotine levels have subsided, nAChR levels in the PFC return to baseline levels. In contrast, at this stage, mGluR2 levels have reduced significantly below baseline levels, thereby altering mGluR2 signaling during short-term plasticity, augmenting spike-timing-dependent potentiation and hampering attention performance. This reduction in mGluR2 signaling underlies the reduced attention performance observed in animals after nicotine exposure during adolescence (Counotte et al., 2011). Thereby, mGluR2 signaling could be a therapeutic target for alleviating attention and impulse control problems in later life.

New questions and opportunities arise from these recent findings. The long-term adaptations involving mGluR2s can have profound implications for network functioning and affect more complex levels of information processing. What are the steps that lead from nicotine exposure and nAChR activation in the adolescent brain to adaptations in synaptic mGluR2 
levels? Unveiling the signaling routes involved may provide a broader view on the adaptation strategies used during brain development in response to environmental factors. Another interesting question would be whether mGluR2 signaling is involved in a broader spectrum of attention impairments with different etiology. If changes in mGluR2 signaling are a common underlying mechanism for attention malfunction it would make it a suitable pharmacological target for therapy.

\section{REFERENCES}

Abreu-Villaca, Y., Seidler, F. J., Qiao, D., Tate, C. A., Cousins, M. M., Thillai, I., and Slotkin, T. A. (2003). Short-term adolescent nicotine exposure has immediate and persistent effects on cholinergic systems: critical periods, patterns of exposure, dose thresholds. Neuropsychopharmacology 28, 1935-1949.

Adriani, W., Spijker, S., DerocheGamonet, V., Laviola, G., Le Moal, M., Smit, A. B., and Piazza, P. V. (2003). Evidence for enhanced neurobehavioral vulnerability to nicotine during periadolescence in rats. J. Neurosci. 23, 4712-4716.

Alkondon, M., and Albuquerque, E. X. (2004). The nicotinic acetylcholine receptor subtypes and their function in the hippocampus and cerebral cortex. Prog. Brain Res. 145, 109-120.

Alkondon, M., Pereira, E. F., Eisenberg, H. M., and Albuquerque, E. X. (2000). Nicotinic receptor activation in human cerebral cortical interneurons: a mechanism for inhibition and disinhibition of neuronal networks. J. Neurosci. 20, 66-75.

Belluzzi, J. D., Lee, A. G., Oliff, H. S., and Leslie, F. M. (2004). Agedependent effects of nicotine on locomotor activity and conditioned place preference in rats. Psychopharmacology (Berl.) 174, 389-395.

Bergstrom, H. C., McDonald, C. G., French, H. T., and Smith, R. F. (2008). Continuous nicotine administration produces selective, age-dependent structural alteration of pyramidal neurons from prelimbic cortex. Synapse 62, 31-39.

Bi, G., and Poo, M. (2001). Synaptic modification by correlated activity: Hebb's postulate revisited. Annu. Rev. Neurosci. 24, 139-166.

Bradley, S. R., Marino, M. J., Wittmann, M., Rouse, S. T., Awad, H., Levey, A. I., and Conn, P. J. (2000). Activation of group II metabotropic glutamate receptors inhibits synaptic excitation of the substantia Nigra pars reticulata. J. Neurosci. 20, 3085-3094.
Brielmaier, J. M., McDonald, C. G., and Smith, R. F. (2007). Immediate and long-term behavioral effects of a single nicotine injection in adolescent and adult rats. Neurotoxicol. Teratol. 29, 74-80.

Brook, J. S., Schuster, E., and Zhang, C. (2004). Cigarette smoking and depressive symptoms: a longitudinal study of adolescents and young adults. Psychol. Rep. 95, 159-166.

Brown, R. A., Lewinsohn, P. M., Seeley, J. R., and Wagner, E. F. (1996). Cigarette smoking, major depression, and other psychiatric disorders among adolescents. J. Am. Acad. Child Adolesc. Psychiatry 35, 1602-1610.

Brown, R. W., and Kolb, B. (2001). Nicotine sensitization increases dendritic length and spine density in the nucleus accumbens and cingulate cortex. Brain Res. 899, 94-100.

Brunzell, D. H., Russell, D. S., and Picciotto, M. R. (2003). In vivo nicotine treatment regulates mesocorticolimbic CREB and ERK signaling in C57Bl/6J mice. J. Neurochem. 84 , 1431-1441.

Casey, B. J., Tottenham, N., Liston, C., and Durston, S. (2005). Imaging the developing brain: what have we learned about cognitive development? Trends Cogn. Sci. 9, 104-110.

Cervilla, J. A., Prince, M., and Mann, A. (2000). Smoking, drinking, and incident cognitive impairment: a cohort community based study included in the Gospel Oak project. J. Neurol. Neurosurg. Psychiatry 68 622-626.

Choi, W. S., Patten, C. A., Gillin, J. C., Kaplan, R. M., and Pierce, J. P. (1997). Cigarette smoking predicts development of depressive symptoms among U.S. adolescents. Ann. Behav. Med. 19, 42-50.

Chung, S., Li, X., and Nelson, S. B. (2002). Short-term depression at thalamocortical synapses contributes to rapid adaptation of cortical sensory responses in vivo. Neuron 34, 437-446.

Conn, P. J., Lindsley, C. W., and Jones, C. K. (2009). Activation

\section{ACKNOWLEDGMENTS}

Huibert D. Mansvelder received funding from the Netherlands Organization for Scientific Research (NWO; 917.76.360 and 912.06.148), European Research Council Starting Grant "BrainSignals," the Dutch Fund for Economic Structure Reinforcement (FES, 0908 "NeuroBasic PharmaPhenomics project"), EU 7th Framework Programme (HEALTH-F2-2009. 242167 "SynSys") and VU University Amsterdam.

of metabotropic glutamate receptors as a novel approach for the treatment of schizophrenia. Trends Pharmacol. Sci. 30, 25-31.

Couey, J. J., Meredith, R. M. Spijker, S., Poorthuis, R. B., Smit, A. B., Brussaard, A. B. and Mansvelder, H. D. (2007). Distributed network actions by nicotine increase the threshold for spike-timing-dependent plasticity in prefrontal cortex. Neuron 54, 73-87.

Counotte, D. S., Goriounova, N. A. Li, K. W., Loos, M., van der Schors, R. C., Schetters, D., Schoffelmeer, A. N. M., Smit, A. B., Mansvelder, H. D., Pattij, T., and Spijker, S. (2011). Lasting synaptic changes underlie attention deficits caused by nicotine exposure during adolescence. Nat. Neurosci. 14 417-419.

Counotte, D. S., Goriounova, N. A. Moretti, M., Smoluch, M. T., Irth, H., Clementi, F., Schoffelmeer, A. N. M., Mansvelder, H. D., Smit, A. B., Gotti, C., and Spijker, S. (2012). Adolescent nicotine exposure transiently increases high-affinity nicotinic receptors and modulates inhibitory synaptic transmission in rat medial prefrontal cortex. FASEB J. 26, 810-820.

Counotte, D. S., Spijker, S., van de Burgwal, L. H., Hogenboom, F., Schoffelmeer, A. N., De Vries, T. J., Smit, A. B., and Pattij, T. (2009). Long-lasting cognitive deficits resulting from adolescent nicotine exposure in rats. Neuropsychopharmacology 34 299-306.

Cruz, D. A., Eggan, S. M., and Lewis, D. A. (2003). Postnatal development of pre- and postsynaptic GABA markers at chandelier cell connections with pyramidal neurons in monkey prefrontal cortex. J. Comp. Neurol. 465, 385-400.

Currie, C. N. G. S., Godeau, E., Roberts, C., Smith, R., Currie, D., Pickett, W., Richter, M., Morgan, A., Barnekow, V. (2008). "Inequalities in young people's health (1993). HBSC international report from the 2005/06
Survey," in Health Policy for Children and Adolescents, No. 5. ed C. Currie (Kopenhagen: WHO Regional Office for Europe).

Dani, J. A., and Bertrand, D. (2007). Nicotinic acetylcholine receptors and nicotinic cholinergic mechanisms of the central nervous system. Annu. Rev. Pharmacol. Toxicol. 47, 699-729.

Deas, D. (2006). Adolescent substance abuse and psychiatric comorbidities. J. Clin. Psychiatry 67(Suppl. 7), $18-23$.

Doura, M. B., Gold, A. B., Keller, A. B. and Perry, D. C. (2008). Adult and periadolescent rats differ in expression of nicotinic cholinergic receptor subtypes and in the response of these subtypes to chronic nicotine exposure. Brain Res. 1215, 40-52.

Feldman, D. E., and Brecht, M. (2005). Map plasticity in somatosensory cortex. Science 310, 810-815.

Feldman, D. E., Nicoll, R. A., and Malenka, R. C. (1999). Synaptic plasticity at thalamocortical synapses in developing rat somatosensory cortex: LTP, LTD and silent synapses. J. Neurobiol. 41, 92-101.

Floresco, S. B., Braaksma, D. N., and Phillips, A. G. (1999). Thalamiccortical-striatal circuitry subserves working memory during delayed responding on a radial arm maze. J. Neurosci. 19,11061-11071.

Fountain, S. B., Rowan, J. D., Kelley, B. M., Willey, A. R., and Nolley, E. P. (2008). Adolescent exposure to nicotine impairs adult serial pattern learning in rats. Exp. Brain Res. 187, 651-656.

Fu, X. W., Lindstrom, J., and Spindel, E. R. (2009). Nicotine activates and up-regulates nicotinic acetylcholine receptors in bronchial epithelial cells. Am. J. Respir. Cell Mol. Biol. 41, 93-99.

Galvan, A., Hare, T., Voss, H., Glover, G., and Casey, B. J. (2007). Risktaking and the adolescent brain: who is at risk? Dev. Sci. 10, F8-F14.

Giedd, J. N. (2004). Structural magnetic resonance imaging of the adolescent brain. Ann. N.Y. Acad. Sci. 1021, 77-85. 
Gioanni, Y., Rougeot, C., Clarke, P. B., Lepouse, C., Thierry, A. M., and Vidal, C. (1999). Nicotinic receptors in the rat prefrontal cortex: increase in glutamate release and facilitation of mediodorsal thalamo-cortical transmission. Eur. J. Neurosci. 11, $18-30$

Gogtay, N., Giedd, J. N., Lusk, L., Hayashi, K. M., Greenstein, D., Vaituzis, A. C., Nugent, T. F. 3rd., Herman, D. H., Clasen, L. S., Toga, A. W., Rapoport, J. L., and Thompson, P. M. (2004). Dynamic mapping of human cortical development during childhood through early adulthood. Proc. Natl. Acad. Sci. U.S.A. 101, 8174-8179.

Goriounova, N. A., and Mansvelder, H. D. (2012). Nicotine exposure during adolescence leads to shortand long-term changes in spiketiming-dependent plasticity in rat prefrontal cortex. J. Neurosci. [Epub ahead of print].

Govind, A. P., Vezina, P., and Green, W. N. (2009). Nicotine-induced upregulation of nicotinic receptors: underlying mechanisms and relevance to nicotine addiction. Biochem. Pharmacol. 78, 756-765.

Grady, S. R., Murphy, K. L., Cao, J., Marks, M. J., McIntosh, J. M., and Collins, A. C. (2002). Characterization of nicotinic agonist-induced [H-3] dopamine release from synaptosomes prepared from four mouse brain regions. J. Pharmacol. Exp. Ther. 301, 651-660.

Grunbaum, J. A., Kann, L., Kinchen, S., Ross, J., Hawkins, J., Lowry, R., Harris, W. A., McManus, T., Chyen, D., and Collins, J. (2004). Youth risk behavior surveillance-United States (2003). MMWR Surveill. Summ. 53, $1-96$.

Guillem, K., Bloem, B., Poorthuis, R. B., Loos, M., Smit, A. B., Maskos, U., Spijker, S., and Mansvelder, H. D. (2011). Nicotinic acetylcholine receptor beta2 subunits in the medial prefrontal cortex control attention. Science 333, 888-891.

Gulledge, A. T., Park, S. B., Kawaguchi, Y., and Stuart, G. J. (2007). Heterogeneity of phasic cholinergic signaling in neocortical neurons. J. Neurophysiol. 97, 2215-2229.

Gupta, D. S., McCullumsmith, R. E., Beneyto, M., Haroutunian, V., Davis, K. L., and Meador-Woodruff, J. H. (2005). Metabotropic glutamate receptor protein expression in the prefrontal cortex and striatum in schizophrenia. Synapse 57, 123-131.

Hebb, D. (1949). The Organization of Behaviour. New York: John Wiley.
Helton, D. R., Tizzano, J. P., Monn, J. A., Schoepp, D. D., and Kallman, M. J. (1997). LY354740, a metabotropic glutamate receptor agonist which ameliorates symptoms of nicotine withdrawal in rats. Neuropharmacology 36, 1511-1516.

Iniguez, S. D., Warren, B. L., Parise, E. M., Alcantara, L. F., Schuh, B., Maffeo, M. L., Manojlovic, Z., and Bolanos-Guzman, C. A. (2008). Nicotine exposure during adolescence induces a depression-like state in adulthood. Neuropsychopharmacology 34, 1609-1624.

Jacobsen, L. K., Krystal, J. H., Mencl, W. E., Westerveld, M., Frost, S. J. and Pugh, K. R. (2005). Effects of smoking and smoking abstinence on cognition in adolescent tobacco smokers. Biol. Psychiatry 57, 56-66.

Jacobsen, L. K., Mencl, W. E., Constable, R. T., Westerveld, M., and Pugh, K. R. (2007). Impact of smoking abstinence on working memory neurocircuitry in adolescent daily tobacco smokers. Psychopharmacology (Berl.) 193, 557-566.

Jay, T. M., and Witter, M. P. (1991). Distribution of hippocampal CA1 and subicular efferents in the prefrontal cortex of the rat studied by means of anterograde transport of Phaseolus vulgaris-leucoagglutinin. J. Comp. Neurol. 313, 574-586.

Ji, D., and Dani, J. A. (2000). Inhibition and disinhibition of pyramidal neurons by activation of nicotinic receptors on hippocampal interneurons. J. Neurophysiol. 83, 2682-2690.

Jones, S., and Yakel, J. L. (1997) Functional nicotinic ACh receptors on interneurones in the rat hippocampus. J. Physiol. 504( $\mathrm{Pt} \mathrm{3),}$ 603-610.

Kalmijn, S., van Boxtel, M. P., Verschuren, M. W., Jolles, J., and Launer, L. J. (2002). Cigarette smoking and alcohol consumption in relation to cognitive performance in middle age. Am. J. Epidemiol. 156, 936-944.

Kapfer, C., Glickfeld, L. L., Atallah, B. V., and Scanziani, M. (2007). Supralinear increase of recurrent inhibition during sparse activity in the somatosensory cortex. Nat. Neurosci. 10, 743-753

Kawaguchi, Y. (1993). Groupings of nonpyramidal and pyramidal cells with specific physiological and morphological characteristics in rat frontal cortex. J. Neurophysiol. 69 416-431.

Kawaguchi, Y., and Kondo, S. (2002). Parvalbumin, somatostatin and cholecystokinin as chemical markers for specific GABAergic interneuron types in the rat frontal cortex. J. Neurocytol. 31, 277-287.

Kawaguchi, Y., and Kubota, Y. (1997) GABAergic cell subtypes and their synaptic connections in rat frontal cortex. Cereb. Cortex 7 476-486.

Kenny, P. J., Gasparini, F., and Markou, A. (2003). Group II metabotropic and alpha-amino-3-hydroxy-5methyl-4-isoxazole propionate (AMPA)/kainate glutamate receptors regulate the deficit in brain reward function associated with nicotine withdrawal in rats J. Pharmacol. Exp. Ther. 306, 1068-1076.

Kenny, P. J., and Markou, A. (2004) The ups and downs of addiction: role of metabotropic glutamate receptors. Trends Pharmacol. Sci. 25 265-272.

Kota, D., Robinson, S. E., and Imad Damaj, M. (2009). Enhanced nicotine reward in adulthood after exposure to nicotine during early adolescence in mice. Biochem. Pharmacol. 78, 873-879.

O'Dell, L. E. (2009). A psychobiological framework of the substrates that mediate nicotine use during adolescence. Neuropharmacology 56(Suppl. 1), 263-278.

Lambe, E. K., Picciotto, M. R., and Aghajanian, G. K. (2003). Nicotine induces glutamate release from thalamocortical terminals in prefrontal cortex. Neuropsychopharmacology 28, 216-225.

Le Novere, N., Corringer, P. J., and Changeux, J. P. (2002). The diversity of subunit composition in nAChRs: evolutionary origins, physiologic and pharmacologic consequences. J. Neurobiol. 53 447-456.

Lester, H. A., Xiao, C., Srinivasan, R., Son, C. D., Miwa, J., Pantoja, R., Banghart, M. R., Dougherty, D. A., Goate, A. M., and Wang, J. C. (2009). Nicotine is a selective pharmacological chaperone of acetylcholine receptor number and stoichiometry. Implications for drug discovery. AAPS J. 11 167-177.

Letzkus, J. J., Kampa, B. M., and Stuart, G. J. (2007). Does spike timing-dependent synaptic plasticity underlie memory formation? Clin. Exp. Pharmacol. Physiol. 34, 1070-1076.

Liechti, M. E., Lhuillier, L., Kaupmann, K., and Markou, A. (2007) Metabotropic glutamate 2/3 receptors in the ventral tegmental area and the nucleus accumbens shell are involved in behaviors relating to nicotine dependence. J. Neurosci. 27, 9077-9085.

Mansvelder, H. D., Keath, J. R., and McGehee, D. S. (2002). Synaptic mechanisms underlie nicotine-induced excitability of brain reward areas. Neuron 33, 905-919.

Markram, H., Toledo-Rodriguez, M., Wang, Y., Gupta, A., Silberberg, G., and $\mathrm{Wu}, \mathrm{C}$. (2004). Interneurons of the neocortical inhibitory system. Nat. Rev. Neurosci. 5, 793-807.

Marks, M. J., McClure-Begley, T. D., Whiteaker, P., Salminen, O., Brown, R. W., Cooper, J., Collins, A. C., and Lindstrom, J. M. (2011). Increased nicotinic acetylcholine receptor protein underlies chronic nicotine-induced up-regulation of nicotinic agonist binding sites in mouse brain. J. Pharmacol. Exp. Ther. 337, 187-200.

Marks, M. J., Pauly, J. R., Gross, S. D., Deneris, E. S., Hermans-Borgmeyer, I., Heinemann, S. F., and Collins, A. C. (1992). Nicotine binding and nicotinic receptor subunit RNA after chronic nicotine treatment. J. Neurosci. 12, 2765-2784.

Mateo, Z., and Porter, J. T. (2007). Group II metabotropic glutamate receptors inhibit glutamate release at thalamocortical synapses in the developing somatosensory cortex. Neuroscience 146, 1062-1072.

McGehee, D. S. (2002). Nicotinic receptors and hippocampal synaptic plasticity ... it's all in the timing. Trends Neurosci. 25, 171

McGehee, D. S., and Role, L. W. (1995). Physiological diversity of nicotinic acetylcholine receptors expressed by vertebrate neurons. Annu. Rev. Physiol. 57, 521-546.

McQuiston, A. R., and Madison, D. V. (1999). Nicotinic receptor activation excites distinct subtypes of interneurons in the rat hippocampus. J. Neurosci. 19 2887-2896.

Melendez, R. I., Gregory, M. L., Bardo, M. T., and Kalivas, P. W (2004). Impoverished rearing environment alters metabotropic glutamate receptor expression and function in the prefrontal cortex. Neuropsychopharmacology 29 1980-1987.

Millar, N. S., and Gotti, C. (2009). Diversity of vertebrate nicotinic acetylcholine receptors. Neuropharmacology 56, 237-246.

Mineur, Y. S., and Picciotto, M. R. (2008). Genetics of nicotinic acetylcholine receptors: relevance to nicotine addiction. Biochem. Pharmacol. 75, 323-333. 
Miwa, J. M., Freedman, R., and Lester, H. A. (2011). Neural systems governed by nicotinic acetylcholine receptors: emerging hypotheses. Neuron 70, 20-33.

Musso, F., Bettermann, F., Vucurevic, G., Stoeter, P., Konrad, A., and Winterer, G. (2007). Smoking impacts on prefrontal attentional network function in young adult brains. Psychopharmacology (Berl.) 191, 159-169.

Orr, D. P., and Ingersoll, G. M. (1995). The contribution of level of cognitive complexity and pubertal timing to behavioral risk in young adolescents. Pediatrics 95, 528-533.

Palucha, A., and Pilc, A. (2005). The involvement of glutamate in the pathophysiology of depression. Drug News Perspect. 18, 262-268.

Peto R., Chen, Z. M., and Boreham, J. (1999). Tobacco-the growing epidemic. Nat. Med. 5, 15-17.

Pilc, A., Chaki, S., Nowak, G., and Witkin, J. M. (2008). Mood disorders: regulation by metabotropic glutamate receptors. Biochem. Pharmacol. 75, 997-1006.

Polesskaya, O. O., Fryxell, K. J., Merchant, A. D., Locklear, L. L., Ker, K. F., McDonald, C. G., Eppolito, A. K., Smith, L. N., Wheeler, T. L., and Smith, R. F. (2007). Nicotine causes age-dependent changes in gene expression in the adolescent female rat brain. Neurotoxicol. Teratol. 29, 126-140.

Poorthuis, R. B., Bloem, B., Schak, B., Wester, J., de Kock, C. P. J., and Mansvelder, H. D. (2012). Layerspecific modulation of the prefrontal cortex by nicotinic acetylcholine receptors. Cereb. Cortex. [Epub ahead of print].

Poorthuis, R. B., Goriounova, N. A., Couey, J. J., and Mansvelder, H. D. (2009). Nicotinic actions on neuronal networks for cognition: general principles and long-term consequences. Biochem. Pharmacol. 78, 668-676.

Portugal, G. S., Wilkinson, D. S., Turner, J. R., Blendy, J. A., and Gould T. J. (2012). Developmental effects of acute, chronic, and withdrawal from chronic nicotine on fear conditioning. Neurobiol. Learn. Mem. 97, 482-494.

Pozzi, L., Baviera, M., Sacchetti, G., Calcagno, E., Balducci, C. Invernizzi, R. W., and Carli, M. (2011). Attention deficit induced by blockade of N-methyl d-aspartate receptors in the prefrontal cortex is associated with enhanced glutamate release and cAMP response element binding protein phosphorylation: role of metabotropic glutamate receptors 2/3. Neuroscience 176, 336-348.

Richards, M., Jarvis, M. J., Thompson, N., and Wadsworth, M. E. (2003). Cigarette smoking and cognitive decline in midlife: evidence from a prospective birth cohort study. Am. J. Public Health 93, 994-998.

Schochet, T. L., Bremer, Q. Z., Brownfield, M. S., Kelley, A. E., and Landry, C. F. (2008). The dendritically targeted protein Dendrin is induced by acute nicotine in cortical regions of adolescent rat brain. Eur. J. Neurosci. 28, 1967-1979.

Schochet, T. L., Kelley, A. E., and Landry, C. F. (2005). Differential expression of arc mRNA and other plasticity-related genes induced by nicotine in adolescent rat forebrain. Neuroscience 135, 285-297.

Shram, M. J., Funk, D., Li, Z., and Le, A. D. (2006). Periadolescent and adult rats respond differently in tests measuring the rewarding and aversive effects of nicotine. Psychopharmacology (Berl.) 186, 201-208.

Silberberg, G., and Markram, H. (2007). Disynaptic inhibition between neocortical pyramidal cells mediated by Martinotti cells. Neuron 53, 735-746.

Song, S., and Abbott, L. F. (2001) Cortical development and remapping through spike timingdependent plasticity. Neuron 32, 339-350.

Sowell, E. R., Peterson, B. S., Thompson, P. M., Welcome, S. E., Henkenius, A. L., and Toga, A. W. (2003). Mapping cortical change across the human life span. Nat. Neurosci. 6, 309-315.

Sowell, E. R., Thompson, P. M., Leonard, C. M., Welcome, S. E., Kan, E., and Toga, A. W. (2004). Longitudinal mapping of cortical thickness and brain growth in normal children. J. Neurosci. 24, 8223-8231.

Spear, L. P. (2000). The adolescent brain and age-related behavioral manifestations. Neurosci. Biobehav. Rev. 24 417-463.

Takahashi, T., Forsythe, I. D. Tsujimoto, T., Barnes-Davies, M., and Onodera, K. (1996). Presynaptic calcium current modulation by a metabotropic glutamate receptor. Science 274, 594-597.

Tierney, P. L., Degenetais, E., Thierry, A. M., Glowinski, J., and Gioanni, Y (2004). Influence of the hippocampus on interneurons of the rat prefrontal cortex. Eur. J. Neurosci. 20, 514-524.

Trauth, J. A., Seidler, F. J., and Slotkin T. A. (2000). An animal model of adolescent nicotine exposure: effects on gene expression and macromolecular constituents in rat brain regions. Brain Res. 867, 29-39.

Vastola, B. J., Douglas, L. A., Varlinskaya, E. I., and Spear, L. P. (2002). Nicotine-induced conditioned place preference in adolescent and adult rats. Physiol. Behav. 77, 107-114.

Wonnacott, S. (1990). The paradox of nicotinic acetylcholine receptor upregulation by nicotine. Trends Pharmacol. Sci. 11, 216-219.

Wonnacott, S., Sidhpura, N., and Balfour, D. J. K. (2005). Nicotine from molecular mechanisms to behaviour. Curr. Opin. Pharmacol. 5,53 .

Woo, T. U., Pucak, M. L., Kye, C. H., Matus, C. V., and Lewis, D. A. (1997). Peripubertal refinement of the intrinsic and associational circuitry in monkey prefrontal cortex. Neuroscience 80, 1149-1158.

Xiang, Z., Huguenard, J. R., and Prince, D. A. (1998). Cholinergic switching within neocortical inhibitory networks. Science 281, 985-988.

Zucker, R. S. (1989). Short-term synaptic plasticity. Annu. Rev. Neurosci. $12,13-31$.

Zucker, R. S., and Regehr, W. G. (2002). Short-term synaptic plasticity. Annu. Rev. Physiol. 64, 355-405.

Conflict of Interest Statement: The authors declare that the research was conducted in the absence of any commercial or financial relationships that could be construed as a potential conflict of interest.

Received: 11 June 2012; accepted: 16 July 2012; published online: 02 August 2012.

Citation: Goriounova NA and Mansvelder HD (2012) Nicotine exposure during adolescence alters the rules for prefrontal cortical synaptic plasticity during adulthood. Front. Syn. Neurosci. 4:3. doi: 10.3389/fnsyn. 2012.00003

Copyright (C) 2012 Goriounova and Mansvelder. This is an open-access article distributed under the terms of the Creative Commons Attribution License, which permits use, distribution and reproduction in other forums, provided the original authors and source are credited and subject to any copyright notices concerning any third-party graphics etc. 